\title{
CORRECTED FOURIER SERIES AND ITS APPLICATION TO FUNCTION APPROXIMATION
}

\author{
QING-HUA ZHANG, SHUIMING CHEN, AND YUANYUAN QU
}

Received 30 November 2003 and in revised form 9 June 2004

Any quasismooth function $f(x)$ in a finite interval $\left[0, x_{0}\right]$, which has only a finite number of finite discontinuities and has only a finite number of extremes, can be approximated by a uniformly convergent Fourier series and a correction function. The correction function consists of algebraic polynomials and Heaviside step functions and is required by the aperiodicity at the endpoints (i.e., $\left.f(0) \neq f\left(x_{0}\right)\right)$ and the finite discontinuities in between. The uniformly convergent Fourier series and the correction function are collectively referred to as the corrected Fourier series. We prove that in order for the $m$ th derivative of the Fourier series to be uniformly convergent, the order of the polynomial need not exceed $(m+1)$. In other words, including the no-more-than- $(m+1)$ polynomial has eliminated the Gibbs phenomenon of the Fourier series until its $m$ th derivative. The corrected Fourier series is then applied to function approximation; the procedures to determine the coefficients of the corrected Fourier series are illustrated in detail using examples.

\section{Introduction}

The theory about the function approximation of finite functions in a finite interval by a Fourier series emerged as early as the nineteenth century [2]. In particular, for any quasismooth function in $\left[0, x_{0}\right]$, which is referred to as the single-valued finite function $f(x)$ that has only a finite number of finite discontinuities as well as a finite number of maxima and minima in the interval $\left[0, x_{0}\right]$, one could obtain the following Fourier series (partial sum):

$$
S_{N}(x)=\sum_{|n| \leq N} A_{n} e^{i \alpha_{n} x}, \quad \alpha_{n}=\frac{2 n \pi}{x_{0}},
$$

where the series coefficient $A_{n}$ is the Fourier projection of $f(x)$ to the basic function $e^{i \alpha_{n} x}$, that is,

$$
A_{n}=\mathscr{F}_{1}\langle f(x)\rangle_{n} \equiv \frac{1}{x_{0}} \int_{0}^{x_{0}} f(x) e^{-i \alpha_{n} x} d x .
$$


Notice that different definitions for smoothness of a function have been used with different meanings in different contexts, for example, in statistics [4]. The concern here is whether the Fourier series (1.1) converges to $f(x)$. The well-known Dirichlet theorem [2] states that for any quasismooth function,

$$
\lim _{N \rightarrow \infty} S_{N}(x)=\lim _{N \rightarrow \infty} \sum_{|n| \leq N} A_{n} e^{i \alpha_{n} x}= \begin{cases}\frac{[f(x-0)+f(x+0)]}{2} & x \in\left(0, x_{0}\right), \\ \frac{\left[f(0)+f\left(x_{0}\right)\right]}{2} & x=0 \text { or } x_{0} .\end{cases}
$$

If $f(x)$ is aperiodic (i.e., $\left.f(0) \neq f\left(x_{0}\right)\right)$ or there are discontinuities in $\left(0, x_{0}\right)$, the Fourier series will not uniformly converge to $f(x)$. The so-called Gibbs phenomenon appears near the endpoints and those discontinuities [3]. On the other hand, if $f(x)$ is a periodic quasismooth function without discontinuities, the Fourier series (1.1) is uniformly convergent to $f(x)$ without the Gibbs phenomenon.

The Gibbs phenomenon is artificial oscillations near the discontinuities and aperiodic endpoints. They are numerical noise without any physical meaning, and should be eliminated if possible. In [1], the Fourier series with Gibbs oscillations is reexpanded into a Gegenbauer series. By doing so, they effectively filter out the Gibbs oscillation and obtain a rapidly convergent series. In this study, we will show that by using a correction function we directly obtain a uniformly convergent Fourier series without Gibbs oscillation (Section 2). We will refer to the correction function and Fourier series as the corrected Fourier series. Since the corrected Fourier series is uniformly convergent, we will apply it to the function approximation, as illustrated in Section 3. Section 4 then provides the concluding remarks.

\section{Corrected Fourier series}

As stated in the introduction, a quasismooth function $f(x)$ in $\left[0, x_{0}\right]$ has only a finite number of finite discontinuities as well as a finite number of maxima and minima in the interval $\left[0, x_{0}\right]$. Relevantly, a quasismooth continuous function is referred to as the quasismooth function without any discontinuity within the interval, but it can be either periodic or aperiodic.

Furthermore, two classes of functions are defined as follows. One is the class of $m$ th quasismooth functions $\mathbf{Q}_{\mathbf{m}}\left(\left[0, x_{0}\right]\right)$, of which the $m$ th derivative of each member is a quasismooth function in $\left[0, x_{0}\right]$. Another is the class of mth quasismooth continuous functions $\mathrm{S}_{\mathrm{m}}\left(\left[0, x_{0}\right]\right)$, of which the $m$ th derivative of each member is a quasismooth continuous function in $\left[0, x_{0}\right]$. Here $m \geq 0$. In the case of $m=0, \mathbf{Q}_{0}\left(\left[0, x_{0}\right]\right)$ has its member being a quasismooth function and $S_{0}\left(\left[0, x_{0}\right]\right)$ being a quasismooth continuous function. The extension of $\mathbf{Q}_{\mathbf{m}}\left(\left[0, x_{0}\right]\right)$ and $\mathbf{S}_{\mathbf{m}}\left(\left[0, x_{0}\right]\right)$ to the cases with multiple variables is straightforward; for two variables they are denoted as $\mathbf{Q}_{\mathbf{m}}\left(\left[0, x_{0}\right],\left[0, y_{0}\right]\right)$ and $\mathbf{S}_{\mathbf{m}}\left(\left[0, x_{0}\right],\left[0, y_{0}\right]\right)$, respectively.

In addition, an mth uniformly convergent Fourier series in an interval means that the Fourier series remains uniformly convergent until its $m$ th derivative without Gibbs phenomenon. 
For clarity, the following notations will be used:

$$
\begin{gathered}
\left.F(x)\right|_{0} ^{x_{0}} \equiv F\left(x_{0}\right)-F(0), \\
\left.\left.F(x, y)\right|_{0} ^{x_{0}}\right|_{0} ^{y_{0}} \equiv F\left(x_{0}, y_{0}\right)+F(0,0)-F\left(x_{0}, 0\right)-F\left(0, y_{0}\right) .
\end{gathered}
$$

Heaviside step function $\mathscr{H}\left(x-x_{j}\right)$ is defined as

$$
\mathscr{H}\left(x-x_{j}\right)= \begin{cases}1, & x \geq x_{j} \\ 0, & x<x_{j}\end{cases}
$$

Lemma 2.1. Any mth quasismooth function $g_{m}(x) \in \mathbf{Q}_{\mathbf{m}}\left(\left[0, x_{0}\right]\right)$ can be partitioned into an mth quasismooth continuous function $f_{m}(x) \in \mathrm{S}_{\mathbf{m}}\left(\left[0, x_{0}\right]\right)$ and $m$ th integrals of a set of Heaviside step functions as follows:

$$
g_{m}(x)=f_{m}(x)+\sum_{j} b_{j} \frac{\left(x-x_{j}\right)^{m}}{m !} \mathscr{H}\left(x-x_{j}\right)
$$

where $x_{j}$ is one of the discontinuities of the mth derivative of $g_{m}(x)$, denoted as $g_{m}^{(m)}(x)$, with jump $b_{j}$, that is, $b_{j}=\left.g_{m}^{(m)}(x)\right|_{x_{j-0}} ^{x_{j+0}}$.

In (2.3), the $m$ th derivative of $f_{m}(x)$, denoted as $f_{m}^{(m)}(x)$, is continuous at the discontinuities of $g_{m}^{(m)}(x)$. This can be easily shown:

$$
\begin{aligned}
\left.f_{m}^{(m)}(x)\right|_{x_{l-0}} ^{x_{l+0}} & =\left.\left[g_{m}^{(m)}(x)-\sum_{j \neq l} b_{j} \mathscr{H}\left(x-x_{j}\right)-b_{l} \mathscr{H}\left(x-x_{l}\right)\right]\right|_{x_{l-0}} ^{x_{l+0}} \\
& =\left.\left[g_{m}^{(m)}(x)-b_{l} \mathscr{H}\left(x-x_{l}\right)\right]\right|_{x_{l-0}} ^{x_{l+0}}=\left.g_{m}^{(m)}(x)\right|_{x_{l-0}} ^{x_{l+0}}-b_{l}=0 .
\end{aligned}
$$

Therefore, $f_{m}(x) \in \mathbf{S}_{\mathbf{m}}\left(\left[0, x_{0}\right]\right)$.

Theorem 2.2. Any mth quasismooth continuous function $f_{m}(x) \in \mathbf{S}_{\mathbf{m}}\left(\left[0, x_{0}\right]\right)$ can be approximated uniformly by the sum of an mth uniformly convergent Fourier series and a polynomial no more than $(m+1)$ th order:

$$
f_{m}(x)=\sum_{|n|<\infty} A_{n} e^{i \alpha_{n} x}+\sum_{l=1}^{m+1} a_{l} \frac{x^{l}}{l !}, \quad \alpha_{n}=\frac{2 n \pi}{x_{0}} .
$$

Proof. We start with $m=0$. Any function $f_{0}(x) \in \mathrm{S}_{\mathbf{0}}\left(\left[0, x_{0}\right]\right)$ is a quasismooth continuous function. It can be expressed by a periodic quasismooth continuous function $h(x)$ and a 
linear function as follows:

$$
f_{0}(x)=h(x)+a x
$$

where $a=\left[f_{0}\left(x_{0}\right)-f_{0}(0)\right] / x_{0}$. It is easy to see the periodicity of $h$ since $h(0)=h\left(x_{0}\right)=$ $f_{0}(0)$. Because $h(x)$ is a periodic, quasismooth continuous function, it can be approximated by a Fourier series that is uniformly convergent:

$$
h(x)=\sum_{|n|<\infty} A_{n} e^{i \alpha_{n} x}, \quad \alpha_{n}=\frac{2 n \pi}{x_{0}},
$$

where

$$
A_{n}=\mathscr{F}_{1}\langle h(x)\rangle_{n}=\frac{1}{x_{0}} \int_{0}^{x_{0}} h(x) e^{-i \alpha_{n} x} d x
$$

It follows that

$$
f_{0}(x)=\sum_{|n|<\infty} A_{n} e^{i \alpha_{n} x}+a x
$$

Notice that the right-hand side of the above equation has two parts: one is the Fourier series and other is a 1st-order polynomial. In other words, the function $f_{0}(x)$ is represented by a corrected Fourier series. Hence, the theorem is true for $m=0$.

In the next step, consider the 1 st quasismooth continuous function $f_{1}(x) \in \mathbf{S}_{\mathbf{1}}\left(\left[0, x_{0}\right]\right)$. Because its first derivative $f_{1}^{(1)}(x)$ is a quasismooth continuous function in the interval $\left[0, x_{0}\right], f_{1}^{(1)}(x)$ can be uniformly approximated by a corrected Fourier series:

$$
f_{1}^{(1)}(x)=\sum_{|n|<\infty} A_{n} e^{i \alpha_{n} x}+a x=\sum_{n \neq 0} A_{n} e^{i \alpha_{n} x}+A_{0}+a x .
$$

Its integration yields

$$
f_{1}(x)=\sum_{n \neq 0} \frac{A_{n}}{i \alpha_{n}} e^{i \alpha_{n} x}+A_{0} x+B_{0}+a \frac{x^{2}}{2 !} \equiv \sum_{|n|<\infty} B_{n} e^{i \alpha_{n} x}+b_{1} x+b_{2} \frac{x^{2}}{2 !},
$$

where

$$
B_{n}=\left\{\begin{array}{ll}
\frac{A_{n}}{i \alpha_{n}} & n \neq 0, \\
B_{0} & n=0,
\end{array} \quad b_{1}=A_{0}, \quad b_{2}=a\right.
$$

Since the termwise integration of a series improves its convergence, the implied uniformly convergence in the above equation is valid. Thus, the theorem is valid for $m=1$.

According to the axiom of the mathematical induction, our proof will be complete if the theorem can be proved true for any $f_{m+1}(x) \in \mathbf{S}_{\mathbf{m + 1}}\left(\left[0, x_{0}\right]\right)$ after it is assumed true for any $f_{m}(x) \in \mathbf{S}_{\mathbf{m}}\left(\left[0, x_{0}\right]\right)$. 
By definition, the 1st derivative of $f_{m+1}(x)$ is an $m$ th quasismooth continuous function in $S_{\mathbf{m}}\left(\left[0, x_{0}\right]\right)$, for which the theorem has been assumed true. Consequently, we have

$$
f_{m+1}^{(1)}(x)=\sum_{|n|<\infty} A_{n} e^{i \alpha_{n} x}+\sum_{l=1}^{m+1} a_{l} \frac{x^{l}}{l !}=\sum_{n \neq 0} A_{n} e^{i \alpha_{n} x}+A_{0}+\sum_{l=1}^{m+1} a_{l} \frac{x^{l}}{l !} .
$$

Its integration yields

$$
\begin{aligned}
f_{m+1}(x) & =\sum_{n \neq 0} \frac{A_{n}}{i \alpha_{n}} e^{i \alpha_{n} x}+A_{0} x+B_{0}+\sum_{l=1}^{m+1} a_{l} \frac{x^{l+1}}{(l+1) !} \\
& =\sum_{n \neq 0} \frac{A_{n}}{i \alpha_{n}} e^{i \alpha_{n} x}+A_{0} x+B_{0}+\sum_{l=2}^{m+2} a_{l-1} \frac{x^{l}}{l !} \\
& =\sum_{|n|<\infty} B_{n} e^{i \alpha_{n} x}+\sum_{l=1}^{m+2} b_{l} \frac{x^{l}}{l !},
\end{aligned}
$$

where

$$
B_{n}=\left\{\begin{array}{ll}
\frac{A_{n}}{i \alpha_{n}} & n \neq 0, \\
B_{0} & n=0,
\end{array} \quad b_{l}= \begin{cases}A_{0} & l=1, \\
a_{l-1} & l \geq 2 .\end{cases}\right.
$$

The right-hand side of (2.14) is the same as that of (2.5). This means that the theorem is true for any $f_{m+1}(x) \in \mathbf{S}_{\mathbf{m}+\mathbf{1}}\left(\left[0, x_{0}\right]\right)$ after it is assumed true for any $f_{m}(x) \in \mathbf{S}_{\mathbf{m}}\left(\left[0, x_{0}\right]\right)$.

Corollary 2.3. Any mth quasismooth function $g_{m}(x) \in \mathbf{Q}_{\mathbf{m}}\left(\left[0, x_{0}\right]\right)$ can be uniformly approximated by a corrected Fourier series consisting of three parts: an mth uniformly convergent Fourier series, a no-more-than $(m+1)$ th-order polynomial, and an mth integral of the Heaviside step functions at the discontinuities

$$
g_{m}(x)=\sum_{|n|<\infty} A_{n} e^{i \alpha_{n} x}+\sum_{l=1}^{m+1} a_{l} \frac{x^{l}}{l !}+\sum_{j} b_{j} \frac{\left(x-x_{j}\right)^{m}}{m !} \mathscr{H}\left(x-x_{j}\right), \quad \alpha_{n}=\frac{2 n \pi}{x_{0}}
$$

It is easily seen that the corollary holds right after Lemma 2.1 and Theorem 2.2.

\section{Function approximation: examples}

As shown in the preceding section, the corrected Fourier series is uniformly convergent. In this section, we will make use of the corrected Fourier series to uniformly approximate quasismooth functions. Two 2 nd quasismooth continuous functions in $\mathbf{S}_{2}\left(\left[0, x_{0}\right]\right)$ and $S_{2}\left(\left[0, x_{0}\right],\left[0, y_{0}\right]\right)$, respectively, have been chosen to show how to determine coefficients of the corrected Fourier series. Extending to higher-order functions should be straightforward but tedious. 
3.1. 2nd quasismooth continuous function $f(x) \in \mathbf{S}_{2}\left(\left[0, x_{0}\right]\right)$. According to the theorem in Section 2, the uniformly convergent corrected Fourier series of $f(x)$ is in the form of

$$
f(x)=\sum_{|n|<\infty} A_{n} e^{i \alpha_{n} x}+a_{1} x+a_{2} \frac{x^{2}}{2 !}+a_{3} \frac{x^{3}}{3 !},
$$

and its 1 st and 2nd derivatives are correspondingly

$$
\begin{gathered}
f^{\prime}(x)=\sum_{|n|<\infty} i \alpha_{n} A_{n} e^{i \alpha_{n} x}+a_{1}+a_{2} x+a_{3} \frac{x^{2}}{2 !}, \\
f^{\prime \prime}(x)=\sum_{|n|<\infty}\left(i \alpha_{n}\right)^{2} A_{n} e^{i \alpha_{n} x}+a_{2}+a_{3} x .
\end{gathered}
$$

As implied, both derivatives of the corrected Fourier series are uniformly convergent.

Based on the endpoints values of $f(x)$ and its derivatives (endpoints effect), we obtain the following linear equations for $a_{l}(l=1,2,3)$ :

$$
\begin{gathered}
\left.f(x)\right|_{0} ^{x_{0}}=a_{1} x_{0}+a_{2} \frac{x_{0}^{2}}{2 !}+a_{3} \frac{x_{0}^{3}}{3 !}, \\
\left.f^{\prime}(x)\right|_{0} ^{x_{0}}=a_{2} x_{0}+a_{3} \frac{x_{0}^{2}}{2 !}, \\
\left.f^{\prime \prime}(x)\right|_{0} ^{x_{0}}=a_{3} x_{0} .
\end{gathered}
$$

Obviously, if $f(x)$ is periodic (i.e., $\left.\left.f(x)\right|_{0} ^{x_{0}}=\left.f^{\prime}(x)\right|_{0} ^{x_{0}}=\left.f^{\prime \prime}(x)\right|_{0} ^{x_{0}}=0\right), a_{l}(l=1,2,3)$ are zero. The corrected Fourier series is just the regular one, which is uniformly convergent in the interval $\left[0, x_{0}\right]$. The coefficients $a_{l}(l=1,2,3)$ are easily obtained by solving (3.3):

$$
\begin{gathered}
a_{3}=\left.\frac{1}{x_{0}} f^{\prime \prime}(x)\right|_{0} ^{x_{0}}, \\
a_{2}=\left.\frac{1}{x_{0}} f^{\prime}(x)\right|_{0} ^{x_{0}}-a_{3} \frac{x_{0}}{2 !}, \\
a_{1}=\left.\frac{1}{x_{0}} f(x)\right|_{0} ^{x_{0}}-a_{2} \frac{x_{0}}{2 !}-a_{3} \frac{x_{0}^{2}}{3 !} .
\end{gathered}
$$

The coefficient $A_{n}$ in (3.1) is the Fourier project (1.2) of $f(x)-\left(a_{1} x+a_{2}\left(x^{2} / 2\right.\right.$ !) + $\left.a_{3}\left(x^{3} / 3 !\right)\right)$ on the basic function $e^{i \alpha_{n} x}$, that is,

$$
A_{n}=\mathscr{F}_{1}\langle f(x)\rangle_{n}-\left(a_{1} I_{1 n}+a_{2} I_{2 n}+a_{3} I_{3 n}\right),
$$

where $I_{l n}=\mathscr{F}_{1}\left\langle x^{l} / l !\right\rangle_{n}$.

If any of $a_{l}(l=1,2,3)$ is nonzero or $f(x)$ is aperiodic, then Gibbs oscillations are expected in $\sum_{n} \mathscr{F}_{1}\langle f(x)\rangle_{n} e^{i \alpha_{n} x}$. However, the same oscillations exist in $\sum_{l=1}^{3} \sum_{n} a_{l} I_{l n} e^{i \alpha_{n} x}$. 
They cancel each other exactly in $\sum_{n} A_{n} e^{i \alpha_{n} x}=\sum_{n} \mathscr{F}_{1}\langle f(x)\rangle_{n} e^{i \alpha_{n} x}-\sum_{l=1}^{3} \sum_{n} a_{l} I_{l n} e^{i \alpha_{n} x}$. As a result, $\sum_{n} A_{n} e^{i \alpha_{n} x}$ is free of Gibbs oscillations and is uniformly convergent.

3.2. 2nd quasismooth continuous function with two unknowns $f(x, y) \in \mathbf{S}_{2}\left(\left[0, x_{0}\right]\right.$, $\left.\left[0, y_{0}\right]\right)$. At first, the function $f(x, y)$ is a 2 nd quasismooth continuous function with respect to $x$. Therefore, for any $y \in\left[0, y_{0}\right]$,

$$
f(x, y)=\sum_{|n|<\infty} A_{n}(y) e^{i \alpha_{n} x}+a_{1}(y) x+a_{2}(y) \frac{x^{2}}{2 !}+a_{3}(y) \frac{x^{3}}{3 !} .
$$

In the above equation, $A_{n}(y)$ and $a_{l}(y)$ are 2 nd quasismooth continuous functions with respect to $y$. Thus, $A_{n}(y)$ and $a_{l}(y)$ can further expand into the following corrected Fourier series:

$$
\begin{aligned}
& A_{n}(y)=\sum_{|m|<\infty} A_{n m} e^{i \beta_{m} y}+b_{1 n} y+b_{2 n} \frac{y^{2}}{2 !}+b_{3 n} \frac{y^{3}}{3 !} \\
& a_{l}(y)=\sum_{|m|<\infty} a_{l m} e^{i \beta_{m} y}+d_{l 1} y+d_{l 2} \frac{y^{2}}{2 !}+d_{l 3} \frac{y^{3}}{3 !} \quad(l=1,2,3)
\end{aligned}
$$

where $\beta_{m}=2 m \pi / y_{0}$. From (3.6) and (3.7), the corrected Fourier series for $f(x, y)$ is

$$
\begin{aligned}
f(x, y)= & \sum_{|n|<\infty} \sum_{|m|<\infty} A_{n m} e^{i\left(\alpha_{n} x+\beta_{m} y\right)}+\sum_{|m|<\infty}\left(a_{1 m} x+a_{2 m} \frac{x^{2}}{2 !}+a_{3 m} \frac{x^{3}}{3 !}\right) e^{i \beta_{m} y} \\
& +\sum_{|n|<\infty}\left(b_{1 n} y+b_{2 n} \frac{y^{2}}{2 !}+b_{3 n} \frac{y^{3}}{3 !}\right) e^{i \alpha_{n} x}+\sum_{l=1}^{3} \sum_{l_{0}=1}^{3} d_{l l_{0}} \cdot \frac{x^{l}}{l !} \cdot \frac{y^{l_{0}}}{l_{0} !} .
\end{aligned}
$$

In (3.8), nine unknowns $d_{l l_{0}}\left(l, l_{0}=1,2,3\right)$ are obtained by solving the following linear equations:

$$
\begin{gathered}
\left.\left.\sum_{l=1}^{3} \sum_{l_{0}=1}^{3} d_{l l_{0}} \cdot \frac{x^{l-j}}{(l-j) !} \cdot \frac{y^{l_{0}-j_{0}}}{\left(l_{0}-j_{0}\right) !} \mathscr{H}(l-j) \mathscr{H}\left(l_{0}-j_{0}\right)\right|_{0} ^{x_{0}}\right|_{0} ^{y_{0}} \\
=\left.\left.\frac{\partial^{j+j_{0}} f(x, y)}{\partial x^{j} \partial y^{j_{0}}}\right|_{0} ^{x_{0}}\right|_{0} ^{y_{0}} \quad\left(j=0,1,2 ; j_{0}=0,1,2\right),
\end{gathered}
$$

which depend on the boundary values of $f(x, y)$ and its 1 st and 2 nd partial derivatives only. Notice that the first three terms on the right-hand side of (3.8) are identically zero due to the periodicity of either $e^{i \alpha_{n} x}$ or $e^{i \beta_{m} y}$. If we arrange the nine unknowns into a vector ordered as $\left(d_{11}, d_{12}, d_{13}, d_{21}, d_{22}, d_{23}, d_{31}, d_{32}, d_{33}\right)$ and the equations ordered as $j=$ $0, j_{0}=0,1,2 ; j=1, j_{0}=0,1,2$; and $j=2, j_{0}=0,1,2$, then the coefficient matrix of the linear equations is up-triangular and can be easily inverted. 
The next task is to determine $a_{l m}, b_{l n}$, and $A_{n m}$. In addition to the Fourier projection $\mathscr{F}_{1}\langle\cdot\rangle_{n}(1.2)$, two additional Fourier projections are defined:

$$
\begin{aligned}
\mathscr{F}_{2}\langle\cdot\rangle_{m} & =\frac{1}{y_{0}} \int_{0}^{y_{0}}\langle\cdot\rangle e^{-i \beta_{m} y} d y, \\
\mathscr{F}_{0}\langle\cdot\rangle_{n m} & =\frac{1}{x_{0} y_{0}} \int_{0}^{y_{0}} \int_{0}^{x_{0}}\langle\cdot\rangle e^{-i\left(\alpha_{n} x+\beta_{m} y\right)} d x d y .
\end{aligned}
$$

With respect to $x$, the endpoints effects of $f(x, y)$ and its derivatives yield

$$
\begin{gathered}
\left.f\right|_{0} ^{x_{0}}=\sum_{|m|<\infty}\left(a_{1 m} x_{0}+a_{2 m} \frac{x_{0}^{2}}{2 !}+a_{3 m} \frac{x_{0}^{3}}{3 !}\right) e^{i \beta_{m} y}+\sum_{l=1}^{3} \sum_{l_{0}=1}^{3} d_{l l_{0}} \cdot \frac{x_{0}^{l}}{l !} \cdot \frac{y_{0}^{l_{0}}}{l_{0} !}, \\
\left.\frac{\partial f}{\partial x}\right|_{0} ^{x_{0}}=\sum_{|m|<\infty}\left(a_{2 m} x_{0}+a_{3 m} \frac{x_{0}^{2}}{2 !}\right) e^{i \beta_{m} y}+\sum_{l=2}^{3} \sum_{l_{0}=1}^{3} d_{l l_{0}} \cdot \frac{x_{0}^{l-1}}{(l-1) !} \cdot \frac{y_{0}^{l_{0}}}{l_{0} !} \\
\left.\frac{\partial^{2} f}{\partial x^{2}}\right|_{0} ^{x_{0}}=\sum_{|m|<\infty}\left(a_{3 m} x_{0}\right) e^{i \beta_{m} y}+\sum_{l_{0}=1}^{3} d_{3 l_{0}} \cdot x_{0} \cdot \frac{y_{0}^{l_{0}}}{l_{0} !} .
\end{gathered}
$$

Notice that the first and third terms in (3.8) are exactly zero due to the periodicity of $e^{i \alpha_{n} x}$. After the Fourier projection to $e^{-i \beta_{m} y}$ (i.e., $\mathscr{F}_{2}\langle\cdot\rangle_{m}$ ), the following equations are resulted and are solved for $a_{l m}(l=1,2,3)$ for each $m$ :

$$
\begin{gathered}
a_{1 m}+a_{2 m} \frac{x_{0}^{2}}{2 !}+a_{3 m} \frac{x_{0}^{3}}{3 !}=\mathscr{F}_{2}\left\langle\left. f\right|_{0} ^{x_{0}}\right\rangle_{m}-\sum_{l=1}^{3} \sum_{l_{0}=1}^{3} d_{l l_{0}} \cdot \frac{x_{0}^{l}}{l !} J_{l_{0} m}, \\
a_{2 m} x_{0}+a_{3 m} \frac{x_{0}^{2}}{2 !}=\mathscr{F}_{2}\left\langle\left.\frac{\partial f}{\partial x}\right|_{0} ^{x_{0}}\right\rangle_{m}-\sum_{l=2}^{3} \sum_{l_{0}=1}^{3} d_{l l_{0}} \cdot \frac{x_{0}^{l-1}}{(l-1) !} J_{l_{0} m}, \\
a_{3 m} x_{0}=\mathscr{F}_{2}\left\langle\left.\frac{\partial^{2} f}{\partial x^{2}}\right|_{0} ^{x_{0}}\right\rangle_{m}-\sum_{l_{0}=1}^{3} d_{3 l_{0}} \cdot x_{0} J_{l_{0} m},
\end{gathered}
$$

where $J_{l_{0} m}=\mathscr{F}_{2}\left\langle y^{l_{0}} / l_{0} !\right\rangle_{m}$.

Similarly, the endpoints effect of $f(x, y)$ and its partial derivative with respect to $y$ result in the following linear equations for $b_{l n}$ for each $n$ :

$$
\begin{gathered}
b_{1 n}+b_{2 n} \frac{y_{0}^{2}}{2 !}+b_{3 n} \frac{y_{0}^{3}}{3 !}=\mathscr{F}_{1}\left\langle\left. f\right|_{0} ^{y_{0}}\right\rangle_{n}-\sum_{l=1}^{3} \sum_{l_{0}=1}^{3} d_{l l_{0}} \cdot \frac{y_{0}^{l_{0}}}{l_{0} !} I_{l n}, \\
b_{2 n} y_{0}+b_{3 n} \frac{y_{0}^{2}}{2 !}=\mathscr{F}_{1}\left\langle\left.\frac{\partial f}{\partial y}\right|_{0} ^{y_{0}}\right\rangle_{n}-\sum_{l=1}^{3} \sum_{l_{0}=2}^{3} d_{l l_{0}} \cdot \frac{y_{0}^{l_{0}-1}}{\left(l_{0}-1\right) !} I_{l n}, \\
b_{3 n} y_{0}=\mathscr{F}_{1}\left\langle\left.\frac{\partial^{2} f}{\partial y^{2}}\right|_{0} ^{y_{0}}\right\rangle_{n}-\sum_{l=1}^{3} d_{l 3} \cdot y_{0} I_{l n},
\end{gathered}
$$

where $I_{l n}=\mathscr{F}_{1}\left\langle x^{l} / l !\right\rangle_{n}$. 
After $d_{l l_{0}}, a_{l m}$, and $b_{l n}$ are obtained, the rest of the coefficients $A_{n m}$ are readily obtained by the following Fourier projection:

$$
A_{n m}=\mathscr{F}_{0}\langle f(x, y)\rangle_{n m}-\left[\sum_{l=1}^{3} a_{l m} I_{l n}+\sum_{l=1}^{3} b_{l n} J_{l m}+\sum_{l=1}^{3} \sum_{l_{0}=1}^{3} d_{l l_{0}} I_{l n} J_{l_{0} m}\right] .
$$

Just as in the first example, any possible Gibbs oscillations associated with the partial sum of the first term in the above equation will be canceled exactly by those in the square bracket.

After all, each coefficient in (3.8) has been determined. This completes the function approximation of $f(x, y)$ by a corrected Fourier series.

\section{Concluding remarks}

Any quasismooth function can be uniformly approximated by a corrected Fourier series, which consists of a uniformly convergent Fourier series and a correction function. The corrected Fourier series is free of the Gibbs phenomenon, although the quasismooth function can be aperiodic and have discontinuities in general.

The correction function consists of algebraic polynomials and Heaviside step functions. The orders of the polynomials are no more than $(m+1)$, demanding that the $m$ th derivative of the corrected Fourier series be uniformly convergent. The corrected Fourier series will not be overconstrained, if the function to be approximated has defined its derivation only until $m$ th order. The solution of an $m$ th-order ordinary or partial differential equation is one such function whose $(m+1)$ derivative is not necessarily defined. Applications of the corrected Fourier series to linear ordinary differential equations with varying coefficients and to linear partial differential equations on irregular region will be the subject of our future studies.

\section{Acknowledgments}

Support from National Natural Science Foundation of China (no. 49736190 and no. 40176008) and Major State Basic Research Development Program of China (no. G1999043800) is gratefully acknowledged. The comments from four anonymous reviewers helped to improve an earlier version of the manuscript.

\section{References}

[1] D. Gottlieb, C.-W. Shu, A. Solomonoff, and H. Vandeven, On the Gibbs phenomenon. I. Recovering exponential accuracy from the Fourier partial sum of a nonperiodic analytic function, J. Comput. Appl. Math. 43 (1992), no. 1-2, 81-98.

[2] M. Kline, Mathematical Thought from Ancient to Modern Times, Oxford University Press, New York, 1972.

[3] C. C. Lin and L. A. Segel, Mathematics Applied to Deterministic Problems in the Natural Sciences, Macmillan Publishing, New York, 1974.

[4] M. Woodroofe, On choosing a delta-sequence, Ann. Math. Statist. 41 (1970), no. 5, 1665-1671. 


\section{Corrected Fourier series and function approximation}

Qing-Hua Zhang: Key Laboratory of Marine Science and Numerical Modeling, The First Institute of Oceanography, State Oceanic Administration, Qingdao 266061, China

E-mail address: zhangqh@fio.org.cn

Shuiming Chen: Key Laboratory of Marine Science and Numerical Modeling, The First Institute of Oceanography, State Oceanic Administration, Qingdao 266061, China

Current address: Department of Oceanography, University of Hawaii at Manoa, 1000 Pope Road, Honolulu, HI 96822, USA

E-mail address: schen@soest.hawaii.edu

Yuanyuan Qu: Key Laboratory of Marine Science and Numerical Modeling, The First Institute of Oceanography, State Oceanic Administration, Qingdao 266061, China

E-mail address: quyy@fio.org.cn 


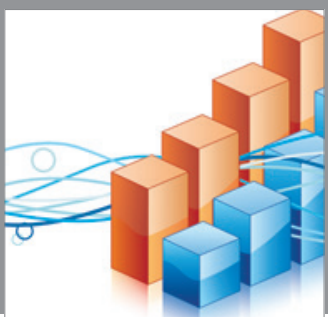

Advances in

Operations Research

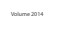

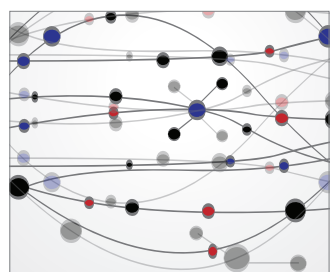

\section{The Scientific} World Journal
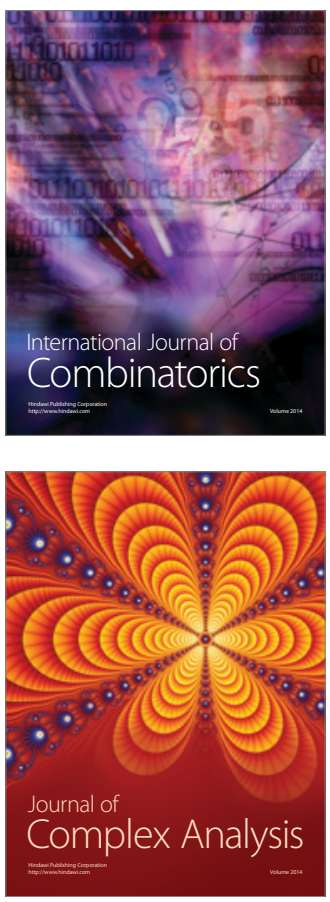

International Journal of

Mathematics and

Mathematical

Sciences
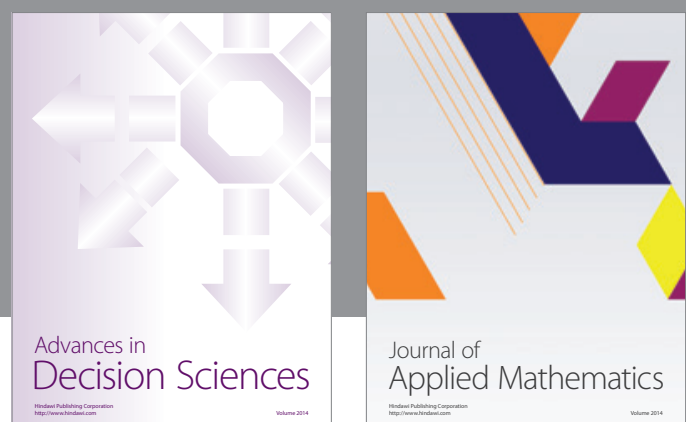

Journal of

Applied Mathematics
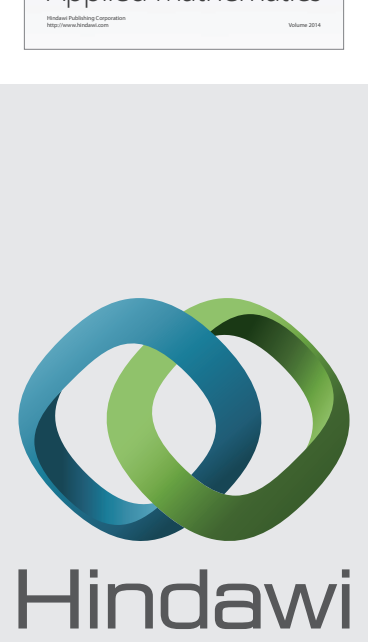

Submit your manuscripts at http://www.hindawi.com
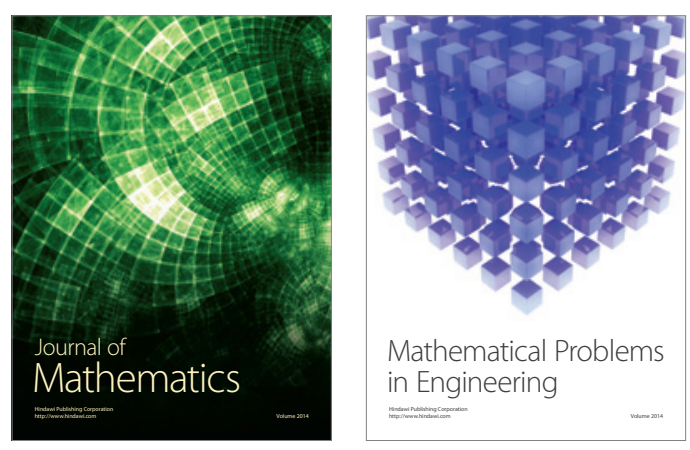

Mathematical Problems in Engineering
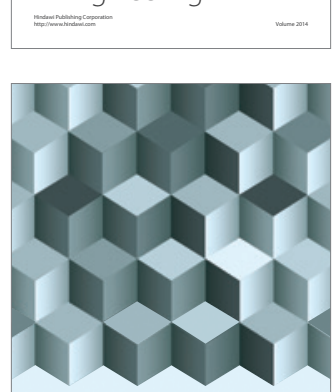

Journal of

Function Spaces
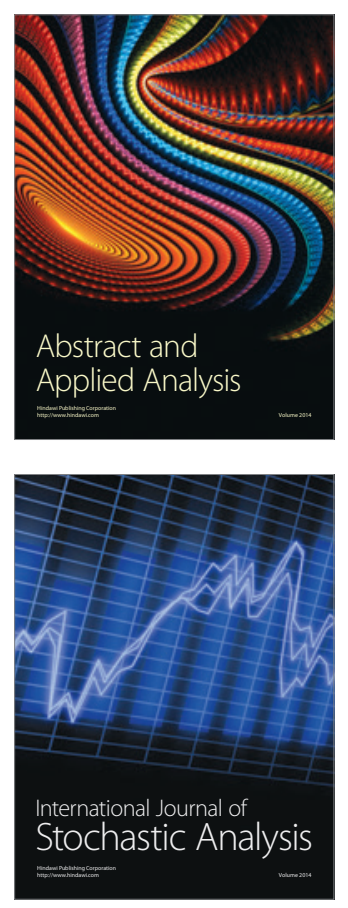

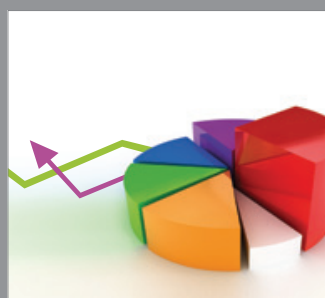

ournal of

Probability and Statistics

Promensencen
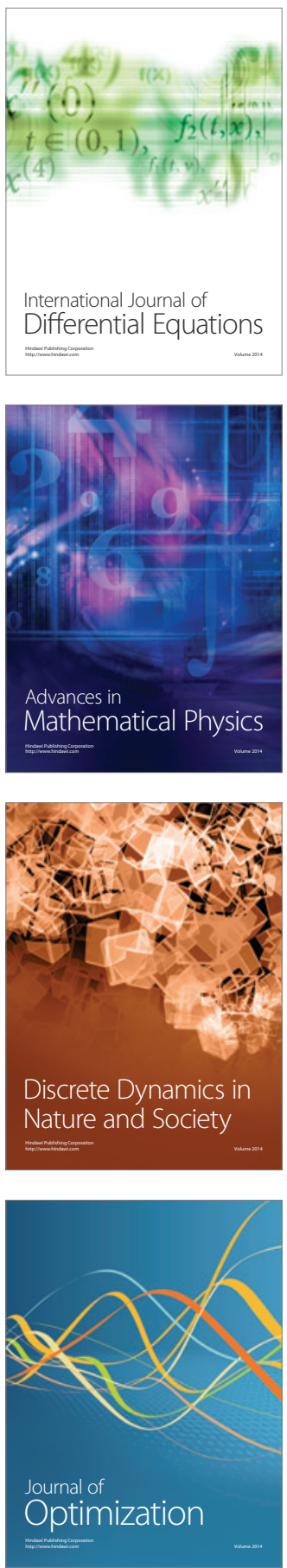